\title{
Guest editorial: current understanding of thrombosis and hemostasis-from bench to bedside
}

\author{
Toshiyuki Miyata
}

Received: 5 March 2012/ Accepted: 6 March 2012/Published online: 5 April 2012

(c) The Japanese Society of Hematology 2012

\section{Introduction}

In this "Progress in Hematology", I would like to offer four excellent invited reviews in the field of thrombosis and hemostasis. On July 23-27, 2011, the XXIII Congress of the International Society on Thrombosis and Haemostasis was held in Kyoto, even after the unprecedented earthquake and tsunami on March 11, followed by the nuclear power plant accident. The Local Organizing Committee hosted more than 160 invited guest speakers and more than 3,000 oral and poster presentations from 75 countries. On this occasion, I had the privilege to organize the "Progress in Hematology" in the fields of thrombosis and hemostasis.

Thrombosis sometimes causes life-threatening events. It is a major healthcare problem and a social burden. Platelet activation and thrombin generation are two key events for thrombosis, which result in the occlusion of the blood vessels and the subsequent ischemic damage to the tissues. Enormous efforts to uncover the detailed molecular mechanisms of thrombus formation have occurred over the last two decades, but the goal is still far. We need to achieve the accumulation of more comprehensive basic, as well as clinical, knowledge of thrombosis. In this regard, basic research in blood coagulation has remained very important for clinical thrombosis. For many years, warfarin and aspirin have been utilized as oral antithrombotic drugs for patients with thrombotic complications and proven to be very effective for the prevention of thrombosis. Heparin is also widely used in clinical settings. In the basic research area, most of the proteins involved in the blood coagulation

T. Miyata $(\bowtie)$

National Cerebral and Cardiovascular Center,

Research Institute, Suita, Japan

e-mail: miyata@ri.ncvc.go.jp have been resolved mainly by crystallography. All of the genes involved in the blood coagulation and its regulation have already been knocked-out in mice, and phenotypes of these mice have been examined intensively in vivo. These progresses link the clinical and the basic approaches to thrombosis. However, there are still mysteries between them, because we do not have enough tools to perfectly regulate thrombosis in vivo. In this "Progress in Hematology", we have four excellent articles on (1) anticoagulant protein $\mathrm{C}$, (2) polyphosphate, a new substance of coagulation initiator and amplifier, (3) von Willebrand factor, and (4) transglutaminase factor XIII.

The first review, by Griffin et al. [1], describes the anticoagulant and cytoprotective functions of activated protein C (APC) and how APC is beneficial for the disease states. APC works as an anticoagulant protein through the proteolytic degradation of activated factor V and VIII, and thereby downregulates the coagulation reactions resulting in the suppression of thrombin generation. APC has another beneficial function, i.e., a cytoprotective function. APC binds to endothelial protein $\mathrm{C}$ receptor EPCR through its Gla-domain and activates protease-activated receptor-1 (PAR-1) through proteolytic cleavage, resulting in generation of the cytoprotective signal. Currently, the APCEPCR-PAR-1 system is a well-recognized agent for antiapoptotic activity, anti-inflammatory activity, endothelial or epithelial barrier protection, and alteration of gene expression profiles. Finally, this review introduces the remarkable neuroprotective effects of APC in vivo using a murine ischemic stroke model.

The review by Morrissey [2] describes novel functions of inorganic polyphosphate in blood coagulation. Polyphosphate, in chains of tens to hundreds of phosphate residues, is abundantly stored in microbes, as well as platelets, and is released by a variety of stimuli. In 
platelets, polyphosphate is stored in dense granules. This polymer provides a polyanionic scaffold to assemble macromolecules including coagulation factors. Biochemical experiments indicated that polyphosphate can initiate the contact phase of coagulation, potentiate the blood coagulation cascade, and modulate the fibrinolytic system. In particular, polyphosphate could most likely be the longsought physiologic activator of factor XII probably released from platelets. In this regard, polyphosphate, by intravenous injection or release from platelets, can induce lethal pulmonary embolism in wildtype mice, but factor XII-deficient mice survived. These in vivo studies demonstrate the physiologic roles of polyphosphate, and polyphosphate is now established as a new modulator of blood coagulation.

The review by Denis and Lenting [3] describes a multimeric plasma protein, von Willebrand factor (VWF). VWF is involved in both hemorrhage and thrombosis. Congenital deficiency in VWF causes a bleeding disorder known as von Willebrand disease. The biological activity of VWF is regulated by the shear force and the multimer size, with the longest being the most active in binding platelets. High molecular weight VWF multimers in blood can be lost by the passage through aortic valve stenosis, and this would result in bleeding. In contrast, epidemiological studies suggested that the increased plasma levels of VWF are a risk for cardiovascular diseases. Unusually large VWF multimers, due to the absence of VWF cleaving protease ADAMTS13, can cause a life-threatening disease, thrombotic thrombocytopenic purpura. In a mouse model, VWF deficiency can protect the focal cerebral ischemia. This review summarizes the various thrombotic disorders related to VWF.

Finally, the review by Ichinose [4] describes recent progress of coagulation factor XIII, a transglutaminase that can crosslink fibrin monomers to make a firm and stable hemostatic plug. Factor XIII consists of two catalytic A subunits and two non-catalytic B subunits and mice lacking these genes have been developed and intensively studied. From the analyses of these mice, the mechanisms of clot retraction have been revealed, and the active involvement of factor XIII in pathogen entrapment through cross-linking of bacteria to fibrin fibers has also been recognized. Congenital deficiency of factor XIII is a rare bleeding disorder. Female patients show recurrent miscarriage. Most of the genetic mutations are found in the $\mathrm{A}$ subunit. Congenital deficiency of the B subunit was also described. Acquired deficiency of factor XIII due to antifactor XIII autoantibody is a rare bleeding disorder. A nationwide study for acquired factor XIII deficiency has started in Japan and patients with factor XIII deficiency are now being identified. Accumulation of the clinical information on these patients will contribute to future improvements of the treatment.

Acknowledgments I would like to thank the authors who took time from their busy schedule to create the excellent reviews and worked on a tight schedule to make the deadline. I thank Dr. Walter Kisiel for the English editing.

\section{References}

1. Griffin JH, Zlokovic BV, Mosnier LO. Protein C anticoagulant and cytoprotective pathways. Int J Hematol. 2012. doi:10.1007/ s12185-012-1059-0.

2. Morrissey JH. Polyphosphate: a link between platelets, coagulation and inflammation. Int J Hematol. 2012. doi:10.1007/s12185-0121054-5.

3. Denis CV, Lenting PJ. von Willebrand factor: at the crossroads of bleeding and thrombosis. Int J Hematol. 2012. doi:10.1007/ s12185-012-1041-x.

4. Ichinose A. Factor XIII as a key molecule at the intersection of coagulation and fibrinolysis as well as inflammation and infection control. Int J Hematol. 2012. doi:10.1007/s12185-012-1064-3. 